\section{$\underset{\substack{\text { hommes } \\ \text { \& migrations }}}{ }$}

\section{Hommes \& migrations}

Revue française de référence sur les dynamiques

migratoires

1277 | 2009

France - Allemagne

\title{
La désignation de soi des personnes d'origine étrangère en France et en Allemagne au début des années 1990
}

\section{Beate Collet}

\section{OpenEdition}

\section{Journals}

\section{Édition électronique}

URL : http://journals.openedition.org/hommesmigrations/167

DOI : 10.4000/hommesmigrations. 167

ISSN : 2262-3353

Éditeur

Musée national de l'histoire de l'immigration

\section{Édition imprimée}

Date de publication : 1 janvier 2009

Pagination : 86-92

ISSN : 1142-852X

\section{Référence électronique}

Beate Collet, « La désignation de soi des personnes d'origine étrangère en France et en Allemagne au début des années $1990 »$, Hommes \& migrations [En ligne], 1277 | 2009, mis en ligne le 29 mai 2013, consulté le 22 avril 2019. URL : http://journals.openedition.org/hommesmigrations/167 ; DOI :

10.4000/hommesmigrations.167 


\section{La désignation de soi des personnes d'origine étrangère en France et en Allemagne au début des années 1990}

Par Beate Collet, sociologue, maître de conférences, université Paris-Sorbonne

À la fin du $X X^{e}$ siècle, les populations issues de l'immigration en Allemagne et en France véhiculent des histoires nationales et juridiques

à la fois différentes et convergentes. Deux enquêtes sociologiques, portant sur des personnes d'origine turque en Allemagne et maghrébine

en France, analysent les rapports entre appartenance culturelle et religieuse dans chaque contexte national. Trois formes identitaires

pour trois modes d'intégration coexistent des deux côtés du Rhin : différenciation, assimilation et participation citoyenne. Elles révèlent l'évolution des identités culturelles et des contextes nationaux récents. 
Malgré un rapprochement de plus en plus évident de l'Allemagne et de la France, dans le cadre de l'Union européenne, le contexte sociétal contemporain de ces deux pays est encore marqué par des histoires politiques et sociales différentes qui se sont sédimentées dans les cadres juridiques et dans les conceptions collectives nationales. Comparer des réalités postmigratoires dans ces deux contextes nationaux est particulièrement fécond pour plusieurs raisons. Les traditions d'immigrationémigration sont différentes. Alors que la France a favorisé depuis le viII siècle l'immigration, l'Allemagne exportait sa population nombreuse vers le Nouveau Monde. La France a toujours combiné l'immigration d'une main-d'ceuvre avec des visées démographiques, l'Allemagne concevait plus exclusivement une migration de main-d'ceuvre impliquant des retours dans les pays d'origine en cas de non-emploi. Du fait de ces différences, les législations sur l'entrée, le séjour et le travail des étrangers ont été pendant longtemps menées de manière différente. La différence juridique la plus marquante concerne les lois régissant l'accès à la nationalité : elles combinent diversemment le droit du sang et le droit du sol. Même si aujourd'hui ces deux cadres législatifs se rapprochent, elles gardent les traces des traditions nationales différentes. Les deux pays ont instauré le double jus soli comme principe : les enfants d'étrangers, eux-mêmes nés dans le pays, acquièrent automatiquement la nationalité française ou allemande à la naissance. Cette réforme législative est toutefois récente en Allemagne (1999), d'autant plus qu'elle est pour le moment toujours couplée avec une obligation de choisir à l'âge adulte entre la nationalité allemande ou celle du pays des parents.

Ces réalités historiques et juridiques ont laissé des traces dans les conceptions collectives contemporaines des nationaux et des étrangers. Ces derniers sont engagés dans des processus identificatoires réciproques qui s'influencent mutuellement. On peut donc penser que les réalités juridiques, historiques et sociales au niveau national influencent leurs manières de s'autodésigner culturellement et de se projeter comme membres de la société allemande ou française.

\section{Deux enquêtes comparatives originales, une typologie pour l'analyse}

Deux enquêtes qualitatives analysant des phénomènes postmigratoires ont été réalisées et construisent explicitement la comparaison de la France et de l'Allemagne. La première, dite enquête mariages mixtes dans la suite du texte, a été réalisée dans le cadre d'une thèse de doctorat ${ }^{(1)}$ entre 1991 et 1993. Cinquante interviews en France et cinquante en Allemagne ont été menées auprès de 
conjoints étrangers de femmes françaises ou allemandes. Ces derniers étaient pour une grande part originaires de Turquie en Allemagne et originaires des pays du Maghreb en France. La seconde, dite enquête conjugalités des descendants par la suite, a été réalisée en 2005 en collabora-

La France a toujours combiné l'immigration d'une main-d'œuvre avec des visées démographiques, l'Allemagne concevait plus exclusivement une migration de main-d'œuvre impliquant des retours dans les pays d'origine en cas de non emploi. tion avec Emmanuelle Santelli $i^{(2)}$ auprès de quarante couples, vingt en France et vingt en Allemagne ${ }^{(3)}$. Cette fois-ci, il s'agissait de descendants d'immigrés maghrébins en France, et de turcs en Allemagne, nés ou ayant grandi dans ces pays pour quelquesuns, en couple mixte pour d'autres.

Chacune de ces enquêtes s'intéressait en quelque sorte au rapport entre une appartenance culturelle et religieuse particulière (celle de la socialisation primaire avant l'immigration ou celle de la socialisation familiale) et l'intégration dans un cadre national prônant une culture majoritaire différente ${ }^{(4)}$. Les résultats montrent comment, dans chaque contexte national, des formes d'intériorisation spécifiques de la condition d'immigré ou de fils-fille de parents immigrés font perdurer des projections identitaires différentes au-delà de la proximité croissante des réalités sociales dans les deux pays.

Trois formes identitaires ont été envisagées : 1) l'intégration par différenciation n'opère pas de distinction entre la dimension citoyenne et culturelle de l'appartenance et construit les références politiques et culturelles en dehors de la société d'installation ; 2) l'intégration par assimilation ne distingue pas non plus ces deux dimensions, mais opère un transfert des références culturelles et nationales dans le pays d'installation ; et 3) l'intégration par participation citoyenne repose sur une distinction entre la dimension citoyenne et la dimension culturelle. Elle transfère les références politiques dans la société d'installation tout en préservant la référence à l'identité culturelle héritée.

Ces trois types sont virtuellement présents dans chaque société. Ils attestent du fait que tous les individus n'ont pas les mêmes modes d'intégration et ils permettent ensuite d'étudier les variations nationales de ces types, sans pour autant globaliser l'expérience vécue des individus outre mesure sous un même mode national. La présentation de ces types est un peu schématique. Ils soulignent de manière idéal-typique des traits saillants au-delà des générations et par delà les pays d'installation ${ }^{(5)}$. 


\section{L'intégration par différenciation}

Dans l'enquête mariages mixtes, les conjoints étrangers proches de l'intégration par différenciation se perçoivent comme immigrés en France, et comme Ausländer en Allemagne. Ils ont au fond une conception d'une identité ethnique qui s'est en quelque sorte réifiée lors du processus d'intégration. En France, ils refusent de participer au modèle national d'intégration, alors qu'en Allemagne leur identité allogène est renforcée par le statut d'Ausländer, qui soutient la bipolarisation de la société allemande en Inländer et Ausländer. Notons qu'immigré et Ausländer ne renvoient pas aux mêmes registres de signifiants : d'un côté il s'agit plus d'un qualificatif social qui atteste de la migration, voire de l'installation ; de l'autre côté, Ausländer marque l'exclusion juridique, même pour des personnes durablement installées en Allemagne. Dans les deux pays toutefois, immigré ou Ausländer marquent la place dans la société comme des personnes n'étant pas égales aux autres, leur participation est conflictuelle, elles sont de ce fait peut-être relativement plus sensibles à la discrimination.

Dans l'enquête conjugalités des descendants, l'intégration par différenciation semble concerner des descendants d'immigrés qui, par leur vécu social, sont très proches des réalités migratoires, notamment à la suite de va-et-vient, lors de leur enfance ou de leur jeunesse, entre le pays d'origine de leurs parents et leur pays d'installation. Les interviewés ont tendance à se percevoir exclusivement par leurs appartenances culturelles et religieuses, et se disent Algériens, Marocains, Tunisiens ou Turcs. Ils sont néanmoins français en France alors qu'ils sont réellement étrangers en Allemagne, n'ayant pas fait la démarche d'acquérir la nationalité allemande (nés avant la réforme du Code de la nationalité). Les interviewés attestent d'un fort sentiment de stigmatisation et n'expriment pas d'intérêt pour la participation politique en France ou en Allemagne.

D'une enquête à l'autre, notons simplement qu'il s'agissait dans l'enquête mariages mixtes d'un cas de figure un peu particulier en France, alors qu'ils étaient légion en Allemagne; dans l'enquête conjugalités des descendants il semble correspondre à un mode d'intégration spécifique dans les deux pays.

\section{L'intégration par assimilation}

Dans l'enquête mariages mixtes, les conjoints étrangers proches de l'intégration par assimilation se perçoivent en France comme des Français, et en Allemagne ils ont tout fait pour être naturalisés allemands. Dans les deux pays, les représentants de 
ce type se distinguent des autres types par leur statut social et professionnel élevé (avocat, cadre, consultant ou médecin). Ils éprouvent quelquefois un sentiment de reconnaissance à l'égard du pays d'immigration et accordent beaucoup d'importance à la participation politique. La différence entre la France et l'Allemagne réside dans le fait qu'en France la déclaration de nationalité a soutenu leur processus d'intégration, alors qu'en Allemagne la naturalisation n'est intervenue qu'après quelques années, souvent après une procédure longue, et elle consacre en quelque sorte leur bonne intégration en Allemagne.

En ce qui concerne l'enquête conjugalités des descendants, les descendants proches de ce type se perçoivent également comme Français ou Allemands. Ils ont souvent été socialisés dans des familles immigrées qui, par leurs modes de vie, étaient déjà très proches des familles françaises ou allemandes. Il s'avère qu'ils sont plus fréquemment en couple mixte avec des Français ou des Allemands non immigrés. Leurs origines maghrébines ou turques sont facilement reléguées au second plan et survivent non pas comme des pratiques culturelles réelles, mais comme des attributs folkloriques ou symboliques. Les descendants d'immigrés proches de ce type favorisent des références sociales liées à leur vie professionnelle ou à leurs activités sportives et associatives. Ils ne cherchent pas à se distinguer des personnes de la société majoritaire. Leurs expériences se distinguent peu dans les deux sociétés, Français de naissance pour les uns, naturalisés allemands pour les autres, ils accordent de l'importance à la participation politique, mais sont globalement peu sensibles aux préoccupations liées à l'immigration.

Alors que l'acquisition de la nationalité française ou allemande faisait la différence pour les conjoints de l'enquête mariages mixtes, leurs spécificités nationales disparaissent progressivement dans l'enquête conjugalités des descendants. S'il persiste une différence entre les discours dans les deux pays, elle réside dans le fait que, dans un cas, ces descendants d'immigrés sont sans conteste français, alors qu'ils doivent se battre pour être allemands dans l'autre. La légitimité de se dire allemand ne semble pas être la même, elle garde encore, chez les jeunes générations issues de l'immigration nées en Allemagne, des allures de revendication.

\section{L'intégration par participation citoyenne}

Dans l'enquête mariages mixtes, les conjoints proches de l'intégration par participation citoyenne se caractérisaient par le fait qu'ils se situent pleinement dans la société française ou allemande, mais qu'ils souhaitent rester fidèles à leurs références culturelles et religieuses. Ils entendent contribuer à une plus grande 
prise en compte des réalités multiculturelles. En France, ils sont Français, du fait de leur mariage, et exercent pleinement leur citoyenneté. En Allemagne en revanche, ils ne sont pas naturalisés, notamment parce qu'ils ne voulaient pas perdre la nationalité turque, mais ceuvraient politiquement pour une reconnaissance de la double appartenance nationale (doppelte Staatsangehörigkeit) en Allemagne. Cette double appartenance revendiquée était souvent aussi appelée double citoyenneté (doppelte Staatsbürgerschaft), sans que ce soit toujours évident s'ils souhaitaient exercer leurs droits politiques à la fois en Allemagne et en Turquie, ou en tant qu'Allemand et Turc en Allemagne. Rétrospectivement, on se rend compte aujourd'hui que le début des années 1990 a été la période à la suite de laquelle le débat sur la nationalité et la citoyenneté a considérablement changé en Allemagne. Progressivement, il est devenu possible de concevoir une citoyenneté fédérale sans fondement ethnique allemand.

En ce qui concerne les descendants d'immigrés de l'enquête conjugalités des descendants, il s'avère qu'ils sont pleinement dans leur citoyenneté française ou allemande sans pour autant abandonner l'héritage culturel légué par leurs parents. Ils tiennent à maintenir des pratiques culturelles et religieuses relevant de l'univers culturel du pays d'origine de leurs parents. Souvent, ils ont privilégié un mariage endogame entre personnes de même origine, pour justement s'assurer d'une bonne entente culturelle et religieuse, et d'une continuité entre la génération de leurs parents et celle de leurs enfants. Il semblerait que ces éléments culturels soient exprimés de manière plus ouverte pour les descendants d'immigrés turcs en Allemagne, que pour les descendants d'immigrés maghrébins en France, notamment en ce qui concerne la transmission de la langue turque. Alors que le discours des interviewés est assez unanime sur leur place et leur avenir dans la société allemande, ce sont des Turcs vivant en Allemagne. En France, ils semblent également tenir à la reconnaissance de leurs spécificités culturelles, mais celles-ci sont plutôt exprimées par le qualificatif de Français d'origine maghrébine.

\section{Turcs en Allemagne, Maghrébins en France}

Cette démonstration montre que les identités culturelles semblent se maintenir assez bien. Elles gardent toute leur importance pour les générations issues de l'immigration, étant nées ou ayant grandi en France et en Allemagne, et ne s'effacent pas vraiment derrière les appartenances nationales majoritaires. Certes, des stigmatisations spécifiques liées au phénotype et au patronyme y contribuent, mais pas seulement. Les références identitaires s'appuient sur des pratiques culturelles 
transmises dans les familles d'origine. Jusqu'à nos jours, on constate les traces d'histoires nationales différentes et d'une difficile transformation de la conception nationale allemande vers une plus grande ouverture à l'égard des populations issues de l'immigration. Or, la conception nationale française est également devant de nouveaux défis. Elle semble être moins en mesure d'intégrer les populations par simple absorption dans la nation française. Cependant, les deux enquêtes révèlent que les sociétés française et allemande ne sont plus les mêmes d'une période à l'autre. Alors qu'il est devenu plus légitime de se reconnaitre dans la citoyenneté allemande quand on a des origines immigrées, il semblerait qu'il soit devenu plus légitime de revendiquer une identité spécifique en France.

\section{Notes}

1. Collet, Beate, Citoyennetés et mariage mixte en France et en Allemagne, thèse de doctorat, EHESS, Paris,1996.

2. Collet, Beate, Santelli, Emmanuelle, Les conjugalités mixtes des descendants d'immigrés en France et en Allemagne : Modes de formation conjugale et dynamiques familiales, Rapport de recherche remis à l'Institut des sciences de l'Homme, Lyon, octobre 2006.

3. Les vingt interviews en Allemagne ont été réalisées grâce à un financement de la Fondation Balzan (Italie/Suisse).

4. Pour l'utilisation du concept d'intégration, cf. Collet, Beate, "Pour l'étude des modes d'intégration entre participation citoyenne et références culturelles", Revue européenne des sciences sociales, tome XLIV, n 135, p. 93-107, 2006.

5. Pour plus de précisions sur la construction de la typologie, cf. Collet, Beate, 2003, "Modes d'intégration nationale et mariage mixte en France et en Allemagne : à propos d'un processus de construction de comparaison internationale", in : Lallement, Michel, Spurk, Jan (dir.), Stratégies de la comparaison internationale, CNRS Éditions, Paris, p. 233-247. 